\title{
The Impact of Seasonal Fluctuations on Rat Liver Mitochondria Response to Tested Compounds- A Comparison between Autumn and Spring. New Insight into Collecting and Interpretation of Experimental Data Originating from Different Seasons
}

\author{
Magdalena Labieniec-Watala ${ }^{1^{*}}$, Karolina Siewiera ${ }^{2}$ \\ ${ }^{1}$ Department of Thermobiology, Faculty of Biology and Environmental Protection, University of Lodz, Lodz, Poland \\ ${ }^{2}$ Department of Haemostasis and Haemostatic Disorders, Medical University of Lodz, \\ University Clinical Hospital No. 2, Lodz, Poland \\ Email: "*magdalab@biol.uni.lodz.pl,ksiewiera@gmail.com
}

Received December 13, 2012; revised January 15, 2013; accepted January 25, 2013

\begin{abstract}
Seasonal variations play an essential role in the metabolism, behavior and activity of the laboratory animals. This study was aimed to examine whether mitochondrial function can be influenced by the seasonal changes and how large is the impact of these fluctuations on experiments with using animal models and further results interpretation. Liver mitochondria were isolated from male Wistar rats and exposed to calcium ions, PAMAM dendrimers G2.5 or their combination: $\left(\mathrm{Ca}^{2+}\right)$ and dendrimer. The scientific hypothesis assumed that dendrimer $\mathrm{G} 2.5$ is able to limit the detrimental effect of $\mathrm{Ca}^{2+}$ ions on mitochondria function, possibly through affecting the following parameters: calcium transport, mitochondrial potential and membrane fluidity. The activity of mitochondria was monitored using fluorescent labels. The changes in calcium transport were detected using Calcium Green 5-N, the mitochondrial membrane potential and membrane fluidity were elucidated using JC-1 and DPH, respectively. The experiments were carried out during the autumn (October/November) or during the spring (May/June). The obtained data emphasize the effect of seasonal differences on liver mitochondria originating from laboratory animals and outline the importance of planning the experiments during the same seasonal period in order to receive objective and reliable results in the future. Finally, it was revealed the neutral effect of G2.5 dendrimer on mitochondria and its inability to protect mitochondria against overload of calcium ions regardless of seasonality. It was also evidenced that liver mitochondria isolated from autumn-derived animals were more sensitive to calcium and/or dendrimer exposure in comparison with mitochondria isolated from animals investigated during the spring.
\end{abstract}

Keywords: Calcium Ions; Fluorescence Measurements; Mitochondrial Function; PAMAM Dendrimer G2.5; Seasonal Variations

\section{Introduction}

Mitochondria are important organelles for every cell as the powerhouse to provide energy for a multitude of cellular processes. They are also considered as the hub of metabolic pathways, primary sources of reactive oxygen species, regulators of apoptosis, and buffers of intracellular calcium. Calcium is a universal intracellular signaling molecule that regulates many pathways critical to cell survival. Under physiological conditions, mitochondrial calcium levels are maintained through calcium cycling between cytosol and matrix through the uniporter and

${ }^{*}$ Corresponding author.
$\mathrm{Na} / \mathrm{Ca}$ exchanger activities. In addition, large amounts of calcium can be found in the form of precipitates in the matrix. Calcium overload occurs when mitochondria are exposed to calcium concentrations exceeding the matrix capacity, and may result in membrane de-energization, ROS production, cytochrome c release, permeability transition phenomenon (mPT) and apoptosis activation [1]. Then, the mitochondrial dysfunction leads to many human maladies, including cardiovascular diseases, neurodegenerative disease, and cancer. Therefore, it is very important to protect mitochondria against detrimental impact of calcium overload.

Polyamidoamine (PAMAM) dendrimers are, by far, 
the best studied of the commercialised and divergently synthesised dendrimers. Typically, these dendrimers are available in full generations (amine terminated) and halfgenerations (carboxylic acid terminated) that are representative of both their size (i.e., diameter in angstroms) and molecular weight. Even at low concentrations, the peripheral amine groups of cationic dendrimers damage cell membranes and lead to cell toxicity. In contrast, anionic dendrimers are much less toxic or even non-toxic and have wider application in medical sciences. Therefore, the attention was focused on using PAMAM G2.5 (Scheme 1) as an agent limiting the detrimental $\mathrm{Ca}^{2+}$ effect on mitochondria.

In 2008 we used for the first time the anionic PAMAM dendrimer of the higher generation (G3.5) to protect mitochondria against calcium overload. Unfortunately, we observed that dendrimer G3.5 used at the concentration above $10 \mu \mathrm{M}$ strongly affected the functionality of rat liver mitochondria. On the other hand, the decreased level of calcium ions added to mitochondria externally was revealed after exposure to G3.5 [2]. This promising observation helped to decide to continue these experiments and to use lower dendrimer's generation at the reduced concentration $(5 \mu \mathrm{M})$. We believed that these "improvements" would lead us to the positive verification of our hypothesis stating that chelating properties of anionic PAMAM dendrimers could be applied also against diseases involving mitochondrial dysfunctions.

Recently, some papers reported that PAMAM dendrimers were able to bind metal ions [3-5]. It was evidenced using different methods that ions can be chelated not only buy dendrimers' terminal groups but also by their internal parts, i.e. by ethylenediamine core [4]. It

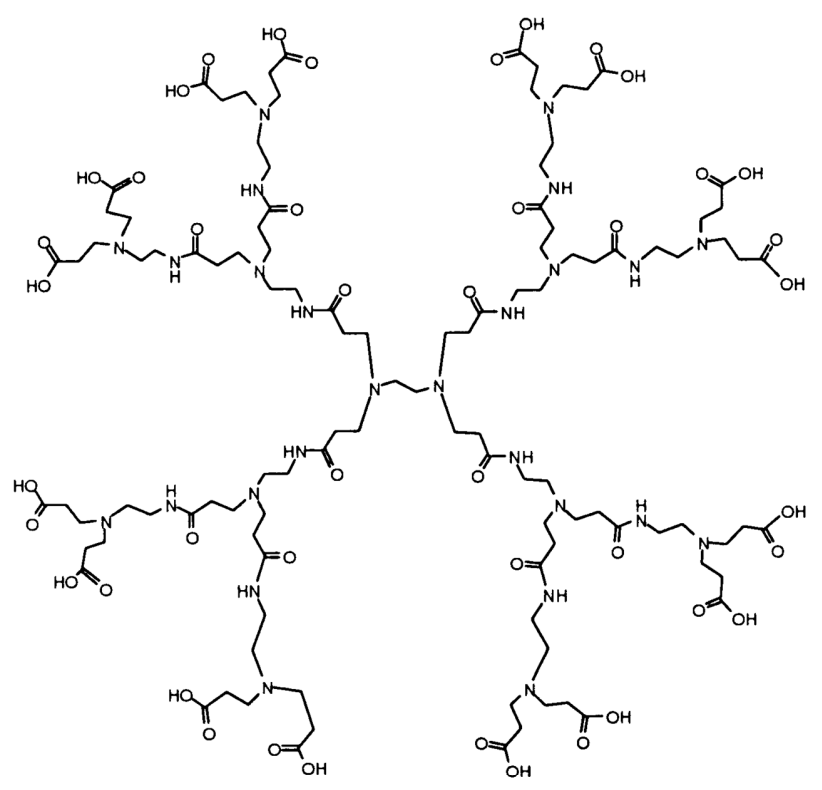

Scheme 1. The structure of PAMAM dendrimer G2.5. was also revealed that anionic dendrimers could form complexes with metals which interact much less strongly with cell biomacromolecules than pure dendrimer or pure metal [5].

Considering the above reports, as well as the other (non referred to herein), the aim of this study was to evaluate the ability of PAMAM G2.5 to bind calcium ions used at the concentrations toxic for rat liver mitochondria. We have chosen to this study only liver mitochondria. First of all, this research is a continuation of our earlier studies on liver mitochondria and anionic PAMAM dendrimer [2]. Secondly, mitochondria isolated from different tissues strongly differ between themselves. Therefore we focused our attention only on one type of mitochondria. Thirdly, liver mitochondria are less sensitive to "environemental changes", i.e. various calcium concentrations in comparison to brain or heart mitochondria. Therefore, in our opinion, the liver mitochondria are a very good model for our study, the main goal of which was to test the hypothesis that PAMAM G2.5 protects the mitochondria against detrimental overload with calcium ions. The calcium-induced mitochondrial changes in some parameters (calcium transport, membrane potential and membrane fluidity) were examined upon exposure to the low concentration of PAMAM dendrimer G2.5, using liver mitochondria isolated from Wistar rats. The exposure of mitochondria to PAMAM dendrimers was conducted in two approaches: 1) without the prior incubation with G2.5 or 2) with 10 min pre-incubation with the dendrimer. The study was performed in four steps. First, we selected the calcium concentration with the highest toxic activity towards the selected mitochondrial parameter, the calcium movement. Second, we evaluated the concentration of PAMAM dendrimer, which appeared neutral (not detrimental) towards another mitochondrial parameter, the transmembrane potential. Third, the activity of G2.5 against the detrimental influence of calcium ions was assessed. Fourth, the impact of seasonal variations (autumn and spring) on the collected data was analyzed.

\section{Materials and Methods}

\subsection{Chemicals}

PAMAM G2.5 dendrimers, $10 \mathrm{wt} \%$ solution in methanol (ethylenediamine core; molecular weight of this dendrimer and the number of terminal sodium carboxylate groups are 6265.60 and 32, respectively), succinate, rotenone, $\mathrm{CaCl}_{2}$, carbonylcyanide m-chlorophenylhydrazone (CCCP), mannitol, EGTA, Tris- $\mathrm{HCl}$, sucrose, Calcium Green 5-N (CG5-N), 5,5',6,6'-tetrachloro-1,1',3,3'tetraethyl benzimidazolyl carbocyanine iodide (JC-1) and 1,6-diphenyl-1,3,5-hexatriene (DPH) were all purchased from Sigma-Aldrich. All other reagents and solvents used in this study were of the highest analytical reagent grade. 


\subsection{Biological Material}

Mitochondria were isolated from livers of Wistar rats (male, 200 - $250 \mathrm{~g}$ ) during autumn (October/November) and spring months (May/June). All animals used for experiment were at the same age and were maintained on the same diet throughout the whole experimental pe$\operatorname{riod}(\mathrm{s})$. Before the experiment, the animals were housed under standard environmental conditions for 2 weeks $\left(25^{\circ} \mathrm{C}\right.$, with a light/dark cycle of $\left.12 \mathrm{~h} / 12 \mathrm{~h}\right)$. The experiments were conducted in accordance with the Guide for the Care and Use of Laboratory Animals published by the US National Institute of Health (NIH Publication No. 85-23, revised 1985), as well as with the guidelines formulated by the European Community for the Use of Experimental Animals (L358-86/609/EEC) and the Guiding Principles in the Use of Animals in Toxicology (1989).

\subsection{Isolation of Liver Mitochondria}

Liver mitochondria were isolated from male Wistar rats according to the procedure described previously [2]. Briefly, liver was removed from the rat killed by decapitation and immediately cooled down to $4^{\circ} \mathrm{C}$ in the homogenization medium ( $75 \mathrm{mM}$ sucrose, $225 \mathrm{mM}$ mannitol, $5 \mathrm{mM}$ Tris- $\mathrm{HCl}, \mathrm{pH} 7.4,0.5 \% \mathrm{BSA}$ and $0.1 \mathrm{M}$ EGTA). The liver tissue was washed to remove blood and connective tissue, cut into small pieces, homogenized using glass homogenizer and centrifuged for $5 \mathrm{~min}$ at $740 \times \mathrm{g}$. Then, the pellet was discarded, and the supernatant was centrifuged again for $10 \mathrm{~min}$ at $9000 \times \mathrm{g}$. The resulting supernatant was removed together with the top layer of the pellet, which contained damaged mitochondria. The dark-brown, "dense" mitochondrial pellet was resuspended in isolation buffer II (225 $\mathrm{mM}$ mannitol, 75

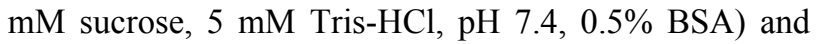
centrifuged again for $10 \mathrm{~min}$ at $10,000 \times \mathrm{g}$. For the final spin $(10,000 \times \mathrm{g}$ for $10 \mathrm{~min})$, the pellet was resuspended in the isolation buffer III (225 mM mannitol, $75 \mathrm{mM}$ sucrose, $5 \mathrm{mM}$ Tris-HCl, $\mathrm{pH}$ 7.4). The mitochondrial pellet was gently resuspended in the isolation buffer (containing $250 \mathrm{mM}$ sucrose, $0.5 \mathrm{mM}$ EDTA, $10 \mathrm{mM}$ Tris and 1 $\mathrm{g} / \mathrm{l}$ bovine serum albumin, $\mathrm{pH}$ 7.4) and stored on ice prior to the experiments.

All isolation procedures were carried out at $0^{\circ} \mathrm{C}-4^{\circ} \mathrm{C}$. During experimentation, mitochondria were stored on ice in the isolation medium until use. The experiments were performed up to $4 \mathrm{~h}$ after preparation. The protein concentration in each sample was determined by the Lowry method.

\subsection{Transport of $\mathrm{Ca}^{2+}$ Ions across Mitochondrial Membranes}

Transmembrane transport of calcium ions following the treatment with PAMAM G2.5 was monitored according to the procedure reported previously [6]. Briefly, the fluorescent dye Calcium Green 5-N was used to record the fluctuations in the extramitochondrial level of calcium ions upon addition of dendrimer G2.5. The whole experiment was divided into 2 stages: 1) the evaluation of $\mathrm{EC}_{50}$ and $\mathrm{EC}_{100}$ for $\mathrm{Ca}^{2+}$ ions (study carried out in autumn) and 2) the estimation of $\mathrm{EC}_{50}$ for dendrimer's activity (the study carried out in spring). Mitochondria (0.3 $\mathrm{mg} / \mathrm{ml}$ protein) were suspended in $1.5 \mathrm{ml}$ of buffer containing $100 \mathrm{mM} \mathrm{KCl}, 10 \mathrm{mM}$ HEPES, $25 \mu \mathrm{M}$ EGTA, 10 $\mu \mathrm{M}$ rotenone, $5 \mathrm{mM}$ succinate, and $200 \mathrm{nM}$ Calcium Green 5-N $\left(\mathrm{K}_{\mathrm{D}}=190 \mathrm{nM}\right), \mathrm{pH}$ 7.2. The following variants were used: 1) $5 \mu \mathrm{M}$ PAMAM G2.5; 2) 10 min preincubation with $5 \mu \mathrm{M}$ PAMAM G2.5; 3) 10 min preincubation with methanol (solvent for dendrimer); and 4) without PAMAM G2.5 (control). The $\mathrm{Ca}^{2+}$ uptake by mitochondria was monitored as the decreased fluorescence of Calcium Green 5-N along with the portions of external $\mathrm{Ca}^{2+}$ added to the medium. A mitochondrial uncoupler, i.e., CCCP (5 $5 \mathrm{M})$, was used for internal control, to obtain maximum depolarization (data not shown), and massive release of $\mathrm{Ca}^{2+}$ by mitochondria in the presence of this protonophore. The titration of the suspension of mitochondria with external calcium led to stepwise $\mathrm{Ca}^{2+}$ accumulation in the medium until calcium overload was achieved. The measurements were conducted with a constant stirring at $37^{\circ} \mathrm{C}$, using a PerkinElmer luminescence spectrometer (Model LS55), and emission continuously recorded at $531 \mathrm{~nm}$ with an excitation wavelength set at $506 \mathrm{~nm}$. The relationships between the concentration of external calcium $(x)$ and the fluorescence intensity of Calcium Green 5-N $(y)$ were fitted to the four parametric regression curves: $y=a+\left\{[b-a] /\left[1+(x / c)^{d}\right]\right\}$, and the values of $\mathrm{EC}_{50}$ (the effective dose of concentration for which the $50 \%$ effect occurs) for the tested samples were calculated by resolving of this function for $\mathrm{f}(x)=y_{\max } / 2$. The coefficients of the above regression curves were iterated with the use of the quasi-Newton algorithm, using the convergence criterion of 0.0001 for $\max 500$ iterations with the initial step of 0.5 for all the coefficients (GraphPad Prism ver. 5 and STATISTICA for Windows, ver. 10).

\subsection{Evaluation of Transmembrane Potential with JC-1}

Mitochondrial transmembrane potential following the treatment with $\mathrm{Ca}^{2+}$ ions and/or PAMAM G2.5 was monitored with the fluorescent cationic dye JC-1 according to the procedure reported by Feeney et al. [7]. The whole experiment was divided into 2 stages: 1) the evaluation of the safe (neutral) concentration of PAMAM dendrimer G2.5 (tested concentrations: 1, 2.5, 5, 10, 20 and $50 \mu \mathrm{M}$; the study carried out in autumn) and 2) the assessment of the activity of the concentration of den- 
drimer selected in the stage 1 (non- and 10-min pre-incubated) with and without $\mathrm{Ca}^{2+}$ ions (used at $\mathrm{EC}_{50}$ and $\mathrm{EC}_{100}$ ), (the study carried out in spring). CCCP $(5 \mu \mathrm{M})$ was used as a positive control (uncoupling compound).

Mitochondria $(0.3 \mathrm{mg} / \mathrm{ml}$ protein $)$ were suspended in $1.5 \mathrm{ml}$ of buffer containing $125 \mathrm{mM} \mathrm{KCl}, 10 \mathrm{mM}$ Tris- $\mathrm{HCl}, 2.5 \mathrm{mM} \mathrm{KH} \mathrm{PO}_{4}, 10 \mathrm{mM}$ EGTA, $10 \mu \mathrm{M}$ rotenone, $5 \mathrm{mM}$ succinate, and $1 \mu \mathrm{M}$ JC-1, $\mathrm{pH}$ 7.4. Fluorescence measurements were performed in a PerkinElmer luminescence spectrometer (Model LS55). The excitation wavelength for JC-1 was $490 \mathrm{~nm}$ and the emission wavelengths were observed at $535 \mathrm{~nm}$ (fluorescence characterised for JC-1 monomers) and at 595 $\mathrm{nm}$ (fluorescence characterized for JC-1 aggregates). Then, the ratio of monomers to aggregates (535/595) was evaluated. The higher membrane depolaryzation, the higher value of this ratio was observed.

\subsection{Assessment of Membrane Fluidity with DPH}

The fluorescence probe DPH (1,6-diphenyl-1,3,5-hexatriene) was used to assess the so-called "fluidity" of the mitochondrial membrane in rat liver mitochondria after the treatment with PAMAM dendrimer G2.5 $(5 \mu \mathrm{M})$ and $\mathrm{Ca}^{2+}$ ions (at $\mathrm{EC}_{50}$ and $\mathrm{EC}_{100}, 37^{\circ} \mathrm{C}$ ) according to the procedure reported previously [6]. Mitochondria were resuspended at a final protein concentration of 0.75 $\mathrm{mg} / \mathrm{ml}$ in the "analysis buffer", containing $60 \mathrm{mM} \mathrm{KCl}$, $10 \mathrm{mM} \mathrm{KH}_{2} \mathrm{PO}_{4}, 60 \mathrm{mM}$ Tris- $\mathrm{HCl}, 0.5 \mathrm{mM}$ EDTA, 5 $\mathrm{mM}$ succinate, and $10 \mu \mathrm{M}$ rotenone, $\mathrm{pH}$ 7.4. Samples were incubated with DPH for 15 min to allow complete incorporation of the probe into the membranes. Fluorescence measurements were performed in a Perkin Elmer luminescence spectrometer (Model LS55). The excitation and emission wavelengths for DPH were selected with monochromators set to $360 \mathrm{~nm}$ (5 nm slit width) and 450 $\mathrm{nm}$ (5 nm slit width), respectively. The degree of fluorescence anisotropy was calculated according to Shinitzky and Barenholz [8].

\subsection{Statistical Analysis}

All measurements of mitochondrial parameters were performed in triplicates. Due to occasional data asymmetry in some variables and groups all data were expressed as median and interquartile lower-upper range $(25 \%$ $75 \%$ ). Data normality was checked using the ShapiroWilk's test and variance homogeneity was verified with Levene's test. Then, data with evidenced normality were analyzed with parametric tests and these with non-proved normality were analyzed with non-parametric tests (MannWhitney U test with Bonferroni's correction for multiple comparisons). For heterogeneous variances, the nonparametric Kruskal-Wallis test and non-parametric Conover-Inman post-hoc test for multiple comparisons were used. The statistical significance between homogenous groups was estimated using one-way ANOVA or twoway ANOVA and post hoc Tukey tests. For all experiments, the number of sample size was estimated for type I and II statistical errors of 0.05 and 0.8 , respectively. Furthermore, the power of used tests was also checked for each (parametric) analysis. The power test below $80 \%$ was considered as unbelievable outcome and the constructive conclusions were not formulated. All statistical calculations were made with the use of STATISTICA.PL v.9 or 10 (StatSoft) and StatsDirect (Stats-Direct Limited). $\mathrm{EC}_{50}$ parameters were calculated using GraphPad Prism (ver. 5).

\section{Results}

All presented results were collected during two different seasonal stages: autumn and spring. We do not show the data from winter or summer. Results obtained in autumn were compared to these ones received in spring and based on these comparisons we have drawn the conclusions presented below. In this paper we report only the part of our study performed on liver mitochondria.

\subsection{Calcium Movements across Mitochondrial Membranes}

The sensitivity of rat liver mitochondria on calcium ions was assessed by fluorescence method, using Calcium Green 5-N for the monitoring of calcium concentration outside the organelles in the suspension of mitochondria. The design of this experiment was to titrate mitochondria suspension with small aliquots of $\mathrm{Ca}^{2+}$ ions (up to $2 \mu \mathrm{M}$ ) and to determine the external concentration of $\mathrm{Ca}^{2+}$ ions, up to which calcium gradually accumulates inside mitochondria until the abrupt depolarization of mitochondrial membrane.

\subsubsection{Effect of $\mathrm{Ca}^{2+}$ Ions on Mitochondria-The Evaluation of $\mathrm{EC}_{50}$ and $\mathrm{EC}_{100}$ during the Autumn Measurements}

Based on the obtained titration curves ( 9 curves from 9 animals), we were able to evaluate the effect of calcium on the "iterated" values of the $\mathrm{EC}_{50}$ and $\mathrm{EC}_{100}$ parameters. The example of such a curve, characteristic for this stage of the study (autumn measurements), was presented in Figure 1. The value of $\mathrm{EC}_{50}$ was $18.5 \pm 1.019 \mu \mathrm{M}$ and $\mathrm{EC}_{100}$ was $37 \pm 2.040 \mu \mathrm{M}$. Data were expressed as mean \pm SEM.

\subsubsection{Effect of PAMAM G2.5 on $\mathrm{Ca}^{2+}$ Mobilization across Mitochondrial Membranes-The Evaluation of $\mathrm{EC}_{50}$ during the Spring Measurements}

In this experiment, the dendrimer G2.5 was used at the 
concentration of $5 \mu \mathrm{M}$ (selected based on the measurements of membrane potential with JC-1). The effect of the dendrimer was tested in two approaches: 1) without the prior pre-incubation and 2) with $10 \mathrm{~min}$ pre-incubation. In Table 1 the values of $\mathrm{EC}_{50}$ are presented, calculated for the autumn and spring measurements.

Based on statistical calculations it was revealed that mitochondria isolated from animals investigated in autumn were more sensitive to calcium ions compared to the organelles isolated from spring animals. As shown in Figure 2, the higher concentration of calcium ions (33.3 $\mu \mathrm{M}$ ) was needed to reach $50 \%$ calcium-induced mitochondria depolarization isolated from spring animals compared to autumn rats $(18.5 \mu \mathrm{M}), \mathrm{P}<0.05$.

The effect of PAMAM dendrimer G2.5 on calcium ions movement across mitochondrial membranes was not observed, neither in the mitochondria not pre-incubated nor in those pre-incubated with PAMAM G2.5. This suggests that dendrimer used at this concentration $(5 \mu \mathrm{M})$ was not able to limit the mitochondrial depolarization caused by $\mathrm{Ca}^{2+}$ ions. On the other hand, the dendrimer did not contribute to the increased sensitivity of mitochondria to calcium, as we evidenced its neutral impact on the tested parameter.

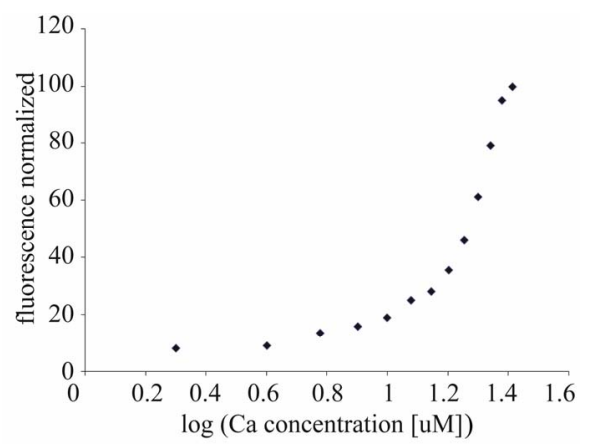

Figure 1. Titration of rat liver mitochondria with calcium ions. The $\mathrm{EC}_{50}$ and $\mathrm{EC}_{100}$ were calculated based on the resolving of the four-parametric curve, $y=a+\{[b-a] /[1+$ $\left.\left.(x / c)^{d}\right]\right\}$, using GraphPad Prism software, ver. 5 for nonlinear regression curves. For further experimental and data analysis details see Section 2.

Table 1. Values of $\mathrm{EC}_{50}$ evaluated in different seasons for rat liver mitochondria exposed to $\mathrm{Ca}^{2+}$ ions and PAMAM G2.5.

\begin{tabular}{|c|c|c|c|c|}
\hline Autumn & & Spring & & $\mathrm{P}$ \\
\hline Control & Control & $+\mathrm{G} 2.5$ & $\begin{array}{c}+\mathrm{G} 2.5 \\
\text { pre-incubated }\end{array}$ & \\
\hline $18.5 \pm 1.0$ & $33.3 \pm 5.5$ & $26.3 \pm 4.6$ & $38.0 \pm 4.5$ & ${ }^{*} 0.0382$ \\
\hline
\end{tabular}

Data are expressed as mean $\pm \mathrm{SEM}, \mathrm{n}=9$ animals (measurements done in triplicates for each animal); $\mathrm{EC}_{50}$ given in $\mu \mathrm{M}$. Statistical significance ${ }^{*} \mathrm{P}=$ 0.0382 ) refers to the comparison of "control" measured in autumn months vs. "control" measured in spring months. The differences for samples measured in spring were not statistically significant: control vs. G2.5, P $=0.6707$; control vs. G2.5 (pre-incubated), $\mathrm{P}=0.9058$ and G2.5 vs. G2.5 (pre-incubated), $\mathrm{P}=0.1556$. For further details see Section 2 .

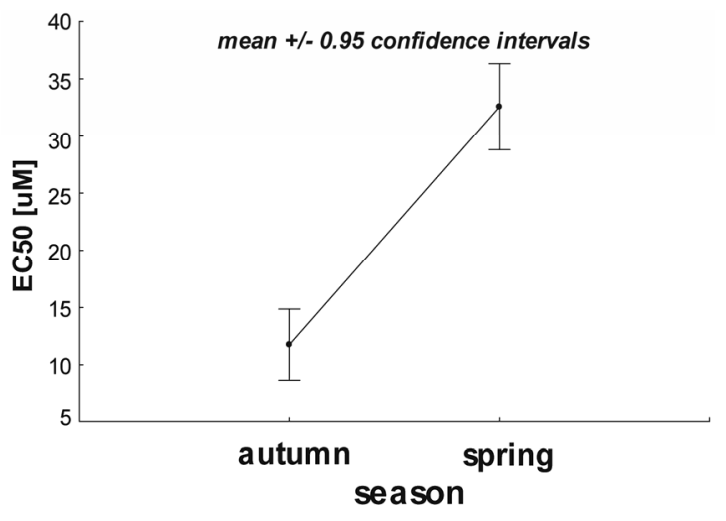

Figure 2. The effect of seasonality on the calcium-mediated rat liver mitochondrial depolarization. Data presented as mean $\pm \mathbf{9 5 \%}$ confidence intervals. Statistical significance the between tested samples (using two-way ANOVA) was $P$ $=0.0382$ (STATISTICA ver. 10, StatSoft). For further experimental and statistical details see Section 2.

\subsection{Transmembrane Potential Evaluation}

Rat liver mitochondrial potential was measured spectrofluorimetrically using JC-1. JC-1 was applied at the concentration of $1 \mu \mathrm{M}$. CCCP $(5 \mu \mathrm{M})$ was used as uncoupler (positive control, data not shown). The interpretation of the mitochondrial potential was based on the changes in the ratio between the fluorescence of JC- 1 monomers and JC-1 aggregates, depending on the changes in mitochondrial potential.

\subsubsection{Effect of PAMAM Dendrimer G2.5 on Mitochondrial Potential-Evaluation of the Neutral Concentration of the Dendrimer- The Autumn Measurements}

The "neutral" concentration of PAMAM G2.5 was chosen based on the changes in mitochondrial potential measured during the autumn period. As shown in Figure 3 , none of the tested G2.5 concentrations $(0,1,2.5,5,10$, 20 and $50 \mu \mathrm{M}$ without preincubation) was shown to significantly affect the mitochondrial potential.

Based on these and other observations (non included in this paper) the concentration of $5 \mu \mathrm{M}$ was selected to be used in further investigations, as completely nontoxic. The significant reduction in mitochondrial potential was observed only for the mitochondria treated with $5 \mu \mathrm{M}$ CCCP (data not shown).

\subsubsection{Effect of PAMAM G2.5 and $\mathrm{Ca}^{2+}$ Ions on Mitochondrial Potential-The Spring Measurements}

The results obtained in this part of the study revealed that the mitochondria studied during the spring period are not sensitive to the tested compounds. Some different approaches were employed: 1) calcium ions used at $\mathrm{EC}_{50}$ $(18.5 \mu \mathrm{M})$ or $\left.\mathrm{EC}_{100}(37 \mu \mathrm{M}) ; 2\right)$ dendrimer $\mathrm{G} 2.5$ used at 


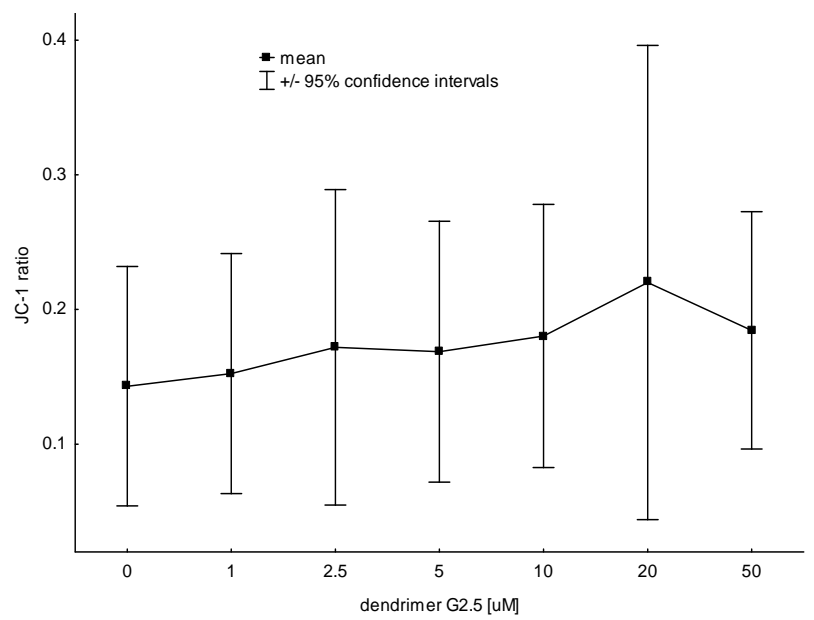

Figure 3. The impact of PAMAM dendrimer G2.5 on rat liver mitochondrial potential, measured with the use of the fluorescence probe, JC-1 (autumn measurements). Data are expressed as mean $\pm 95 \%$ confidence intervals, $n=6$ animals (for each animal 3 mitochondrial preparations were tested). No statistically significant differences were observed between the tested concentrations of the dendrimer. For further experimental and statistical details see Section 2.

$5 \mu \mathrm{M}$ (mitochondria not pre-incubated and pre-incubated for $10 \mathrm{~min}$ with the dendrimer); and 3 ) the combinations of G2.5 and calcium ions used at both the concentrations. As shown in Table 2, calcium used at $\mathrm{EC}_{50}$ and $\mathrm{EC}_{100}$ did not cause any changes in the mitochondrial potential.

No alterations were also observed for dendrimers and the combinations of dendrimer $+\mathrm{Ca}^{2+}$ with the respect to control samples (mitochondria alone). It means that all the tested variants of both the agents had no impact on mitochondrial potential. However, the statistically significant differences were revealed between: 1$)$ the higher $(37 \mu \mathrm{M})$ and the lower $(18.5 \mu \mathrm{M})$ calcium concentration $(\mathrm{P}<0.05)$, as well as between 2$)$ the combination of " 5 $\mu \mathrm{M}$ G2.5 + $37 \mu \mathrm{M} \mathrm{Ca}^{2+}$ ", and "5 $\mu \mathrm{M}$ G2.5" $(\mathrm{P}<0.05)$. These data suggest that there was a tendency to reduce the mitochondrial potential by the higher $\mathrm{Ca}^{2+}$ concentrations, although we did not observe the statistically significant depolarization of mitochondria exposed to 37 $\mu \mathrm{M} \mathrm{Ca}^{2+}$ (non-significant versus control mitochondria).

Interestingly, it was noticed that there was a significant difference in the responses to $\mathrm{G} 2.5(5 \mu \mathrm{M})$ exposure between the "autumn" mitochondria and the "spring" mitochondria. As shown in Figure 4, the "autumn" mitochondria appeared more sensitive (the higher ratio of JC-1 means the reduction in the mitochondrial potential) to G2.5 activity in comparison with the "spring" mitochondria $(P=0.0094)$. Nevertheless, the changes in the potential between the control "autumn" mitochondria and the control "spring" mitochondria were not revealed $(\mathrm{P}=$ $0.0681)$.
Table 2. The effect of PAMAM G2.5 and $\mathrm{Ca}^{2+}$ ions on rat liver mitochondrial potential- the spring evaluation.

\begin{tabular}{|c|c|c|}
\hline Sample & JC-1 ratio & $P$ value \\
\hline Control & $0.036 \pm 0.003$ & \\
\hline $18 \mu \mathrm{M} \mathrm{Ca}^{2+}$ & $0.025 \pm 0.002$ & ns vs. control \\
\hline $37 \mu \mathrm{M} \mathrm{Ca}^{2+}$ & $0.046 \pm 0.006$ & $\begin{array}{c}\text { ns vs. control } \\
0.011 \text { vs. } 18 \mu \mathrm{M} \\
\mathrm{Ca}^{2+}\end{array}$ \\
\hline $5 \mu \mathrm{M} \mathrm{G} 2.5$ & $0.027 \pm 0.002$ & ns vs. control \\
\hline $\begin{array}{c}5 \mu \mathrm{M} \mathrm{G} 2.5 \\
\text { (10 min pre-incubated with } \\
\text { mitochondria) }\end{array}$ & $0.029 \pm 0.002$ & ns vs. control \\
\hline $5 \mu \mathrm{M} \mathrm{G} 2.5+18 \mu \mathrm{M} \mathrm{Ca}^{2+}$ & $0.028 \pm 0.002$ & ns vs. control \\
\hline $\begin{array}{c}5 \mu \mathrm{M} \mathrm{G} 2.5 \text { (pre-incubated) } \\
+18 \mu \mathrm{M} \mathrm{Ca}^{2+}\end{array}$ & $0.032 \pm 0.002$ & ns vs. control \\
\hline $5 \mu \mathrm{M} \mathrm{G} 2.5+37 \mu \mathrm{M} \mathrm{Ca}^{2+}$ & $0.060 \pm 0.013$ & $\begin{array}{c}\text { ns vs. control } \\
0.027 \text { vs. } 5 \mu \mathrm{M} \\
\text { G2.5 }\end{array}$ \\
\hline
\end{tabular}

Data (JC-1 ratio of monomers to aggregates) are presented as mean $\pm \mathrm{SEM}$, $\mathrm{n}=6$ animals (the measurements were done in triplicates for each animal); ns-non statistically significant. For further experimental and statistical details see Section 2.

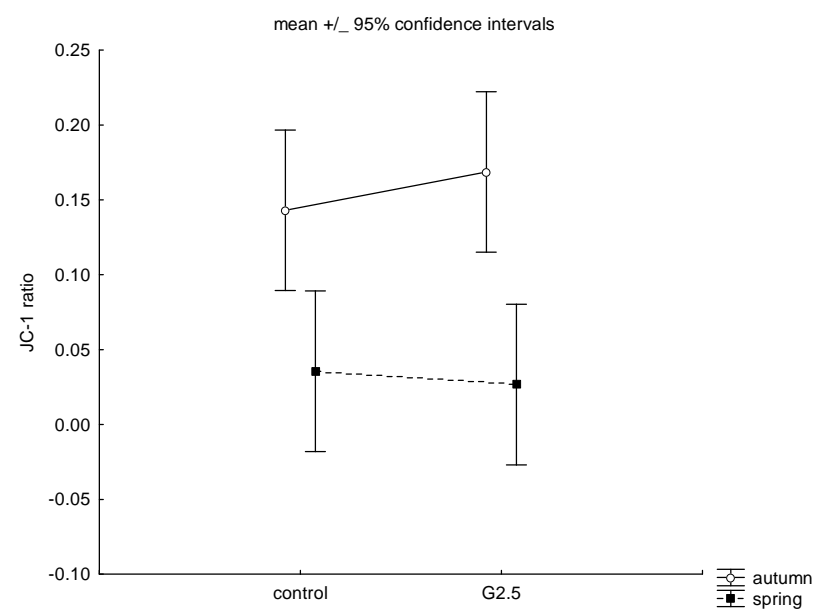

Figure 4. The effect of seasonality (autumn and spring) on PAMAM dendrimer G2.5 (5 $\mu \mathrm{M})$ activity. Data (JC-1 ratio of monomers to aggregates) are presented as mean $\pm 95 \%$ confidence intervals, $n=6$ animals (measurements were done in triplicates for each animal). The statistical significance (two-way ANOVA, StatSoft, ver. 10) was evidenced only between the treatment with PAMAM G2.5 during autumn and spring $(\mathrm{P}<0.005)$. For further experimental and statistical details see Section 2.

Moreover, it was evidenced that the time plays an important role in the mitochondrial potential studies. All tested variants of samples measured after 30 min incubation (room temperature in dark) showed depolarization of mitochondrial membrane at the level of statistically significant, i.e. the JC-1 ratio for control mitochondria in " 0 time" and control mitochondria after "30 min incubation" 
increased from $0.036 \pm 0.003$ to $0.094 \pm 0.006$ (mean \pm SEM), $\mathrm{P}<0.0005$ (the complete data not shown). These results suggest that mitochondria are aging rapidly and it is important to take into account the "passage of time" as a meaningful factor, when measuring mitochondrial potential.

\subsubsection{Mitochondrial Membrane Fluidity Evaluation-The Spring Measurements}

Rat liver membrane fluidity was evaluated based on the anisotropy fluorescence of DPH. The tested compounds were studied according to the design presented in Table 3. The obtained results revealed that none of the applied compounds (or their combinations) had an impact on membrane fluidity. Nevertheless, the power of the performed comparisons was relatively low (below $70 \%$ or lower). Therefore, we have to consider the results of these experiments with a caution. The presented data notwithstanding, are intriguing and certainly deserve to be further explored in the future. Similar phenomena were evidenced for the same samples measured after 30 min of incubation at room temperature: membrane fluidity remained unchanged upon the treatment, and the power of test was below $40 \%$.

\section{Discussion}

In the present study, the hypothesis that PAMAM dendrimer G2.5 protects mitochondria against the overload with calcium ions was tested. It is widely considered that the activity of $\mathrm{Ca}^{2+}$ ions towards mitochondria is ambiguous. On one hand, there are known advantageous effects of calcium, i.e. stimulation of ATP production,

Table 3. The effect of PAMAM G2.5 and $\mathrm{Ca}^{2+}$ ions on rat liver mitochondrial fluidity - the spring evaluation.

\begin{tabular}{|c|c|c|c|}
\hline Sample & $\begin{array}{l}\text { DPH anisotropy } \\
\text { (a.u.) }\end{array}$ & $\begin{array}{c}\mathrm{P} \\
\text { value }\end{array}$ & $\begin{array}{c}\text { Power of } \\
\text { test }[\%]\end{array}$ \\
\hline control & $0.384 \pm 0.004$ & - & - \\
\hline $18 \mu \mathrm{M} \mathrm{Ca}^{2+}$ & $0.395 \pm 0.002$ & ns & 66 \\
\hline $37 \mu \mathrm{M} \mathrm{Ca}^{2+}$ & $0.393 \pm 0.003$ & ns & 10 \\
\hline $5 \mu \mathrm{M} \mathrm{G} 2.5$ & $0.382 \pm 0.004$ & ns & 60 \\
\hline $\begin{array}{c}5 \mu \mathrm{M} \mathrm{G} 2.5 \\
\text { (10 min pre-incubated } \\
\text { with mitochondria) }\end{array}$ & $0.391 \pm 0.005$ & ns & 14 \\
\hline $5 \mu \mathrm{M} \mathrm{G} 2.5+18 \mu \mathrm{M} \mathrm{Ca}^{2+}$ & $0.389 \pm 0.006$ & ns & 12 \\
\hline $\begin{array}{c}5 \mu \mathrm{M} \mathrm{G} 2.5(\text { pre- } \\
\text { incubated })+18 \mu \mathrm{M} \mathrm{Ca}^{2+}\end{array}$ & $0.383 \pm 0.005$ & ns & 5 \\
\hline $5 \mu \mathrm{M} \mathrm{G} 2.5+37 \mu \mathrm{M} \mathrm{Ca}^{2+}$ & $0.381 \pm 0.005$ & ns & 60 \\
\hline
\end{tabular}

Data (DPH fluorescence anisotropy) are presented as mean \pm SEM, $\mathrm{n}=6$ animals (measurements were done in triplicates for each animal); ns-difference non statistically significant. Statistical analysis and power of test were calculated using STATISTICA ver. 9 (StatSoft). Power of test was measured for the comparisons in relation to control. For further experimental and statistical details see Section 2. coordination of cell metabolism or activation of mitochondrial enzymes. On the other hand, in contrast to this beneficial activity, the pathological effect of calcium on mitochondria function has been well evidenced [9]. It cannot be ignored that $\mathrm{Ca}^{2+}$, particularly at high concentrations, appears to have several negative impacts on mitochondria, i.e. induction of $\mathrm{mPT}$ pores, stimulation of mitochondrial uncoupling, activation of apoptosis via mitochondria or interruption of cellular signaling. Therefore, it has become important to find compounds/agents, which would work as efficient modulators of the excessive calcium in the various cellular compartments, including mitochondria.

For a few years our attention has been focused on anionic PAMAM dendrimers. It has been well demonstrated that PAMAM dendrimers are successfully used for many in vitro and in vivo biomedical applications [10]. On the other side, PAMAM dendrimers were tested for their effects on cellular cytotoxicity, lysosomal $\mathrm{pH}$, and mitochondria dependent apoptosis. Some results suggest that full generations of PAMAM dendrimers are endocytosed into the cells through a lysosomal pathway, leading to lysosomal alkalinization and induction of mitochondriamediated apoptosis [11]. Other experimental data also prove the detrimental modulatory effects of cationic dendrimres (especially G4 and higher) on mitochondria function [12-14]. Nevertheless, there are only few literature data concerning the negative influence of half-generations of PAMAMs on mitochondria. Our previous study [2] suggested that, on one hand, PAMAM G3.5 used above $10 \mu \mathrm{M}$ affected the selected rat liver mitochondrial parameters (i.e. caused membrane depolarization), but on the other effectively hindered the influx of $\mathrm{Ca}^{2+}$ to mitochondria. Thus, PAMAM dendrimer G3.5 did not have the desired biological activity, however, our other findings, based upon still unpublished data, suggest that smaller generations and/or smaller concentrations of these molecules should be more effective in the protection of mitochondria against the overload with calcium ions.

Our present data show that PAMAM G2.5, used in a concentration of $5 \mu \mathrm{M}$, does not affect the rat liver mitochondrial parameters, such as membrane potential, membrane fluidity and calcium transport across the membrane. On the other hand, the protective role of this dendrimer (towards mitochondria) was not observed as it was expected at the stage of the formulation of research hypothesis. Maybe, the used concentration of dendrimer was too low, but some data (not shown here) revealed that the concentration of G2.5 above $7.5 \mu \mathrm{M}$ significantly caused the disturbations in mitochondrial bioenergetics (measurements done using the high-fidelity Oroboros-2k oxygraph). Therefore, it was safer to use lower G2.5 concentration when testing its activity. The main assumption 
of this study was based on the proven chelating properties of half-generations PAMAM dendrimers [3-5]. We believed that G2.5 is able to successfully bind excess of $\mathrm{Ca}^{2+}$ ions and thus limits their input to mitochondria. However, it was important to meet two experimental conditions in order to confirm these assumptions. The first one concerned the choice of the neutral concentration of G2.5 towards mitochondrial function and the second one concerned the ability of binding calcium ions by this dendrimer. Neutral concentration of G2.5 was assessed experimentally in our laboratory, but we did not check its chelating activity towards $\mathrm{Ca}^{2+}$. Based only on the literature data, we hoped that even low generations of PAMAMs can effectively bind metal ions, such as: $\mathrm{Ag}^{+}$, $\mathrm{Cu}^{2+}, \mathrm{Zn}^{2+}, \mathrm{Fe}^{2+}$, and $\mathrm{Fe}^{3+}[5,15]$. Nevertheless, so far, the investigation of binding $\mathrm{Ca}^{2+}$ by PAMAM dendrimers was not proven using appropriate experimental techniques (i.e. mass spectrometry). Based on the above information, we need to emphasize that this study has been designed simple examination of the relationship of the "concentration-effect", rather than the proving of $\mathrm{Ca}^{2+}$ chelating properties of G2.5.

Interestingly, we have noticed, although not for the first time $[16,17]$, that the response of mitochondria to the treatment with various compounds depends on the seasonality. The results presented in this paper also indicate that there is a statistically significant difference between the activities of the mitochondria isolated from rats investigated in autumn (October-November) and the mitochondria isolated from animals studied in spring (May-June). Based on the activity of mitochondrial parameters, such as mitochondrial potential and $\mathrm{Ca}^{2+}$ movement across the mitochondrial transmembranes, it appears that "autumn" mitochondria are more sensitive in comparison with "spring" mitochondria. The mitochondrial potential decrease (the higher depolarization) and the resistance to the input of calcium ions overload were observed. Not many laboratories, which conduct the studies with using rats as their animal model, distinguished the impact of seasonal variations on the changes observed in the cell or tissue at the molecular level. So far, some reports relate to the changes in the breeding activity and animal metabolism [18], others refer to the differences in immune system in birds [19], food-catching activity [20] or reproduction behavior [21]. Nevertheless, the recent study of Konior et al., demonstrated that guinea-pig and rat hearts generate more super oxide anion, and human subjects excrete more 8-isoprostane in the summer compared to other seasons [22]. In contrary to our observations, the conclusions mentioned above indicate that the increase of oxidative stress was characteristic for animals studied in summer in comparison to changes observed for animals tested in winter months. On the other hand, it was evidenced in young rats that their arteries were protected from thrombogenesis and lipid accumulation during summer months vs. winter period [23]. The study of Mock and Frankel showed that older rats ( 5 - 6 months old) might be more sensitive to seasonal influences than younger rats (4 months old). Their results indicate that male laboratory rats exhibited seasonality in several reproductive parameters, even though the animals were maintained under constant laboratory conditions [24]. There are a lot of similar examples of studies demonstrating the role of seasonality, which makes us to believe that the influence of the seasons should always be considered when designing of the study. Otherwise, the consequences of carrying out the studies during the whole year without blocking of the obtained data according to the employed laboratory conditions can deliver misleading outcomes. Unfortunately, at this stage of our research, we are not able to explain at biochemical level (i.e. the monitoring of liver glycogen content) why mitochondria differ physiologically, when studied in different seasons. Until now there is a lack of information concerning seasonal influence on the laboratory animals, and thus, more studies are needed to answer questions raised also in this paper.

Despite the strong evidence linking the calcium ions and mitochondria damage, the precise concentration of $\mathrm{Ca}^{2+}$ needed to cause mitochondrial changes remains unknown. It seems likely to be dependent on the source of mitochondria, cellular metabolism and other cellular conditions. Some data showed that $\mathrm{Ca}^{2+}$ used at $10 \mu \mathrm{M}$ caused irreversible and very fast membrane depolarization of rat liver mitochondria [25]. Also the study of Vergun and Reynolds revealed that depolarization of rat liver mitochondria required the use of calcium at $20 \mu \mathrm{M}$ after ca. $10 \mathrm{~min}$ incubation with mitochondria [26]. The same concentration of $\mathrm{Ca}^{2+}$ ions was used to reduce the effectiveness of oxidative phosphorylation in rat liver mitochondria [27]. Taking into account these data it seems that our result- $18.5 \mu \mathrm{M}\left(\mathrm{EC}_{50}\right.$ for $\mathrm{Ca}^{2+}$, assessed during autumn) is in agreement with other literature findings. Surprisingly however, we were not able to obtain the similar results for the same experiment carried out a few months later (spring period). The $\mathrm{EC}_{50}$ for calcium ions was $33.3 \mu \mathrm{M}$, which indicated that the tested mitochondria were much less sensitive to detrimental effects of calcium ions. Probably, the main reason of this differentiation in the received data lies in the distinct seasons (autumn/spring), during which these measurements were done. Consequently, when conducting of the experiments in spring, and using the values of $\mathrm{Ca}^{2+}$ and G2.5 evaluated in autumn, we might have received the data, which could not properly verify the hypothesis. It was very hard to check the protective activity of G2.5 towards mitochondria, which were treated by calcium ions used at the concentration much lower than that 
needed to observe the desired changes. Therefore, in fact, we could only test the impact of PAMAM dendrimer on mitochondrial parameters. Nevertheless, without repeating of the study related to the finding of both non-cytotoxic and cytotoxic concentrations of PAMAM G2.5 also for the spring season, it was hard or even impossible to observe any effect of dendrimer tested at $5 \mu \mathrm{M}$ on mitochondria, regardless of $10 \mathrm{~min}$ pre-incubation of G2.5 with mitochondria before the measurements. In support of this notion, no impact of G2.5 was observed on calcium transport, mitochondrial potential and membrane fluidity, thus suggesting that the used G2.5 concentration probably was too low for spring mitochondria under the tested conditions. Additionally, the experiment with JC-1 using PAMAM G2.5 in the range of concentrations: 1 $50 \mu \mathrm{M}$ and rat liver mitochondria carried out in summer (August) showed that none of the tested concentrations had the impact of mitochondrial potential (data not shown).

In addition to assessing of the main mitochondrial parameters, the effect of mitochondrial "aging", based on the results obtained from membrane potential evaluations, has been studied. It was proven that the same mitochondrial samples revealed statistically significant membrane depolarization measured after 30 min post-incubation (in dark and room temperature). It was clearly evidenced that the passage of time plays an important role during mitochondrial measurements. Nevertheless, it is not a new discovery and mitochondrial functional impairment with aging was evidenced earlier [28,29].

Summarizing, the present data revealed that PAMAM dendrimer G2.5, used at the concentration of $5 \mu \mathrm{M}$ (with and without pre-incuabtion), had no impact on rat liver mitochondrial functions. On the other hand, however, it was proven that it might be too low concentration in order to use it as a regulator of calcium ions in the mitochondrial therapy. These results indicate that in the future the dendrimer G2.5 could be used successfully in further mitochondrial examinations not only in a combination with calcium ions. Nevertheless, all experiments should be finished during the short interval of time in order to limit the influence of seasonal changes on the acquired data.

\section{Conclusion}

The primary objective of this study was the using of PAMAM G2.5 as a regulator of detrimental effect of calcium ions on the main mitochondrial functions. The goal was to find the destructive concentration of calcium ions and the neutral concentration of G2.5 achieved with the use of liver mitochondria isolated from Wistar rats in different seasonal periods (autumn and spring). Based on the obtained results it is worth of noting that seasonal fluctuations have an important effect on measured mito- chondrial parameters. Furthermore, the unsuitable experimental design and their inappropriate statistical analysis (e.g. not including block designed ANOVA) often leads to the misleading conclusions. Therefore, to determine the targeting experimental tools and therapeutic agents to mitochondria with using animals in different age or derived in different seasons requires to demonstrate the great care and knowledge about the entire research model. With greater understanding of physiological differences between tested samples/animals and advances in biomaterial design, there is an opportunity to develop systems that respond to physiological stimuli, seasonal fluctuations as well as the potential to use compounds/ chemicals as promising $\operatorname{drug}(\mathrm{s})$. Use of safe and effective multi-functional nano-platforms promises to alleviate many of the mitochondrial disturbances what may be benefit for treatment of diseases involving mitochondrial disorders in the future.

\section{Acknowledgements}

This work has been funded by grant from Ministry of Science and Higher Education, N N405 261037. Authors thank Anna Jarosz for her excellent technical support. Authors are also grateful to Prof. Cezary Watala for the opportunity to use the laboratory of the Department of Haemostasis and Haemostatic Disorders (Medical University of Lodz) during the collecting of data for this study.

\section{REFERENCES}

[1] H. Kawamata, G. Manfredi, "Mitochondrial Dysfunction and Intracellular Calcium Dysregulation in ALS," Mechanism of Ageing and Development, Vol. 131, No. 7-8, 2010, pp. 517-526. doi:10.1016/j.mad.2010.05.003

[2] M. Labieniec and T. Gabryelak, "Preliminary Biological Evaluation of Poli(Amidoamine) (PAMAM) Dendrimer G3.5 on Selected Parameters of Rat Liver Mitochondria," Mitochondrion, Vol. 8, No. 4, 2008, pp. 305-312. doi:10.1016/j.mito.2008.07.001

[3] S. M. Cohen, S. Petoud and K. N. Raymond, "Synthesis and Metal Binding Properties of Salicylate-, Catecholate-, and Hydroxypyridinonate-Functionalized Dendrimers," Chemistry - A European Journal, Vol. 7, No. 1, 2001, pp. 272-279.

doi:10.1002/1521-3765(20010105)7:1<272::AID-CHEM 272>3.0.CO;2-Y

[4] M. S. Diallo, S. Christie, P. Swaminathan, L. Balogh, X. Shi, W. Um, C. Papelis, W. A. Goddard III and J. H. Johnson Jr., "Dendritic Chelating Agents. 1. Cu(II) Binding to Ethylene Diamine Core Poly(Amidoamine) Dendrimers in Aqueous Solutions," Lanqmuir, Vol. 20, No. 7, 2004, pp. 2640-2651. doi:10.1021/la036108k

[5] S. Sekowski, A. Kazmierczak, J. Mazur, M. Przybyszewska and T. Gabryelak, "The Interaction between PAMAM G3.5 Dendrimer, $\mathrm{Cd}^{2+}$, Dendrimer- $\mathrm{Cd}^{2+}$ Complexes and $\mathrm{Hu}-$ 
man Serum Albumin," Colloids and Surfaces B: Biointerfaces, Vol. 69, No. 1, 2009, pp. 95-98. doi:10.1016/i.colsurfb.2008.11.006

[6] M. Labieniec, T. Przygodzki, J. Carsky, D. Malinska, J. Rysz and C. Watala, "Effects of Resorcylidene Aminoguanidine (RAG) on Selected Parameters of Isolated Rat Liver Mitochondria," Chemico-Biological Interactions, Vol. 179, No. 2-3, 2009, pp. 280-287. doi:10.1016/j.cbi.2008.11.005

[7] Ch. J. Feeney, P. S. Pennefather and A. V. Gyulkhandanyan, "A Cuvette-Based Fluorometric Analysis of Mitochondrial Membrane Potential Measured in Cultured Astrocyte Monolayers," Journal of Neuroscience Methods, Vol. 125, No. 1-2, 2003, pp. 13-25. doi:10.1016/S0165-0270(03)00027-X

[8] M. Shinitzky, "Membrane Fluidity and Cellular Functions," In: M. Shinitzky, Ed., Physiology of Membrane Fluidity, Vol. 12, Plenum Press, New York, 1984, pp. 1-51. doi:10.1007/978-1-4684-4667-8_20

[9] P. S. Brookes, Y. Yoon, J. L. Robotham, M. W. Anders and S.-S. Sheu, "Calcium, ATP, and ROS: A Mitochondrial Love-Hate," American Journal of Physiology: Cell Physiology, Vol. 287, No. 4, 2004, pp. C817-C833.

[10] I. J. Majoros, Ch. R. Williams, D. A. Tomalia and J. R. Baker Jr., "New Dendrimers: Synthesis and Characterization of Popam-Pamam Hybrid Dendrimers," Macromolecules, Vol. 41, No. 22, 2008, pp. 8372-8379. doi:10.1021/ma801843a

[11] T. P. Thomas, I. Majoros, A. Kotlyar, D. Mullen, M. M. Holl and J. R. Baker Jr., "Cationic Poly(Amidoamine) Dendrimer Induces Lysosomal Apoptotic Pathway at Therapeutically Relevant Concentrations," Biomacromolecules, Vol. 10, No. 12, 2009, pp. 3207-3214. doi:10.1021/bm900683r

[12] J. H. Lee, K. E. Cha, M. S. Kim, H. W. Hong, D. J. Chung, G. Ryu and H. Myung, "Nanosized Polyamidoamine (PAMAM) Dendrimer-Induced Apoptosis Mediated by Mitochondrial Dysfunction," Toxicology Letters, Vol. 190, No. 2, 2009, pp. 202-207. doi:10.1016/j.toxlet.2009.07.018

[13] M. Labieniec, O. Ulicna, O. Vancova, J. Kucharska, T. Gabryelak and C. Watała, "Effect of Poly(Amido)Amine (PAMAM) G4 Dendrimer on Heart and Liver Mitochondria in an Animal Model of Diabetes," Cell Biology International, Vol. 34, No. 1, 2010, pp. 89-97.

[14] S. P. Mukherjee, F. M. Lyng, A. Garcia, M. Davoren and H. J. Byrne, "Mechanistic Studies of in Vitro Cytotoxicity of Poly(Amidoamine) Dendrimers in Mammalian Cells," Toxicology and Applied Pharmacology, Vol. 248, No. 3, 2010, pp. 259-268. doi:10.1016/j.taap.2010.08.016

[15] M. A. Kaczorowska and H. J. Cooper, "Electron Capture Dissociation and Collision-Induced Dissociation of Metal Ion $\left(\mathrm{Ag}^{+}, \mathrm{Cu}^{2+}, \mathrm{Zn}^{2+}, \mathrm{Fe}^{2+}\right.$, and $\left.\mathrm{Fe}^{3+}\right)$ Complexes of Polyamidoamine (PAMAM) Dendrimers," Journal of the American Society for Mass Spectrometry, Vol. 20, No. 4, 2009, pp. 674-681. doi:10.1016/j.jasms.2008.12.013

[16] M. Labieniec-Watala, K. Siewiera and Z. Jozwiak, "Resorcylidene Aminoguanidine (RAG) Improve Cardiac Mitochondrial Bioenergetics Impaired by Hyperglycae- mia in a Model of Experimental Diabetes," International Journal of Molecular Sciences, Vol. 12, No. 11, 2011, pp. 8013-8026. doi:10.3390/ijms12118013

[17] K. Siewiera and M. Labieniec-Watala, "Ambiguous Effect of Dendrimer PAMAM G3 on Rat Heart Respiration in a Model of an Experimental Diabetes-Objective Causes of Laboratory Misfortune or Unpredictable G3 Activity?" International Journal of Pharmaceutics, Vol. 430, No. 1-2, 2012, pp. 258-265.

[18] M. L. Carras, E. Brenowitz and E. W. Rubel, "Peripheral Auditory Processing Changes Seasonally in Gambel's White-Crowned Sparrow," Journal of Comparative Physiology A: Neuroethology Sensory, Neural and Behavioral Physiology, Vol. 196, No. 8, 2010, pp. 581-599. doi:10.1007/s00359-010-0545-1

[19] L. B. Martin, Z. M. Weil and R. J. Nelson, "Seasonal Changes in Vertebrate Immune Activity: Mediation by Physiological Trade-Offs," Philosophical Transactions of the Royal Society B: Biological Sciences, Vol. 363, No. 1490, 2008, pp. 321-339. doi:10.1098/rstb.2007.2142

[20] M. T. Avey, A. Rodriguez and Ch. B. Sturdy, "Seasonal Variation of Vocal Behaviour in a Temperate Songbird: Assessing the Effects of Laboratory Housing on WildCaught, Seasonally Breeding Birds," Behavioural Processes, Vol. 88, No. 3, 2011, pp. 177-183. doi:10.1016/j.beproc.2011.09.005

[21] B. J. Prendergast, A. Kampf-Lassin, J. R. Yess, J. Galang, N. McMaster and L. M. Kay, "Winter Lengths Enhance T Lymphocyte Phenotypes, Inhibit Cytokine Responses, and Attenuate Behavioral Symptoms of Infection in Laboratory Rats," Brain Behavior, and Immunity, Vol. 21, No. 8, 2007, pp. 1096-1108. doi:10.1016/j.bbi.2007.05.004

[22] A. Konior, E. Klemenska, M. Brudek, E. Podolecka, E. Czarnowska and A. Beręsewicz, "Seasonal Superoxide Overproduction and Endothelial Activation in Guinea-Pig Heart; Seasonal Oxidative Stress in Rats and Humans," Journal of Molecular and Cellular Cardiology, Vol. 50, No. 4, 2011, pp. 686-694. doi:10.1016/j.yjmcc.2010.11.010

[23] S. Masumura, F. Furui, M. Hashimoto and Y. Watanabe, "The Effects of Season and Exercise on the Levels of Plasma Polyunsaturated Fatty Acids and Lipoprotein Cholesterol in Young Rats," Biochimica and Biophysica Acta: Lipids and Lipid Metabolism, Vol. 1125, No. 3, 1992, pp. 292-296.

[24] E. J. Mock and A. I. Frankel, "A Seasonal Influence on Tests Weight and Serum Gonadotropin Levels of the Mature Male Laboratory Rat," Biology Reproduction, Vol. 18, No. 5, 1978, pp. 772-778. doi:10.1095/biolreprod18.5.772

[25] M. J. Devinney, L. M. Malaiyandi, O. Vergun, D. B. DeFranco, T. G. Hastings and K. E. Dineley, "A Comparison of $\mathrm{Zn}^{2+}$ - and $\mathrm{Ca}^{2+}$-Triggered Depolarization of Liver Mitochondria Reveals No Evidence of $\mathrm{Zn}^{2+}$-Induced Permeability Transition," Cell Calcium, Vol. 45, No. 5, 2001, pp. 447-455. doi:10.1016/j.ceca.2009.03.002

[26] O. Vergun and I. Reynolds, "Distinct Characteristics of $\mathrm{Ca}^{2+}$-Induced Depolarization of Isolated Brain and Liver Mitochondria," Biochimica and Biophysica Acta: Bio- 
energetics, Vol. 1709. No. 2, 2005, pp. 127-137.

[27] P. M. Silva, E. Tanabe, A. P. Hermoso, C. A. BersaniAmado, A. Bracht, E. L. Ishii-Iwamoto and C. L. Salgueiro-Pagadigorria, "Changes in Calcium-Dependent Membrane Permeability Properties in Mitochondria of Livers from Arthritic Rats," Cell Biochemistry and Function, Vol. 26, No. 4, 2008, pp. 443-450. doi: $10.1002 /$ cbf. 1461

[28] M. Picard, D. Ritchie and K. J. Wright, "Mitochondrial Functional Impairment with Aging Is Exaggerated in Iso- lated Mitochondria Compared to Permeabilized Myofibers," Aging Cell, Vol. 9, No. 6, 2010, pp. 1032-1046. doi:10.1111/j.1474-9726.2010.00628.x

[29] M. Picard, D. Ritchie, M. M. Thomas, K. J. Wright and R. T. Hepple, "Alterations in Intrinsic Mitochondrial Function with Aging Are Fiber Type-Specific and Do Not Explain Differential Atrophy between Muscles," Aging Cell, Vol. 10, No. 6, 2011, pp. 1047-1055. doi:10.1111/j.1474-9726.2011.00745.x

\section{Abbreviations}

PAMAM dendrimer: poly(amido)amine dendrimer; mPT pore: mitochondrial permeability transition pore. 